\title{
Hormônios e o "aprimoramento natural" do corpo: a personalização do processo de envelhecimento na medicina anti-aging
} Hormones and the "natural enhancement" of the body: the personalization of aging in anti-aging medicine

\section{Fernanda Rougemont}

(1) https://orcid.org/0000-0003-4971-7232

E-mail: fernandarougemont $\bowtie$ gmail.com

Universidade Federal do Rio de Janeiro. Programa de Planejamento Energético. Instituto Virtual Internacional de Mudanças Globais. Rio de Janeiro, RJ, Brasil.

\section{Correspondência}

Av. Pedro Calmon, s/n, prédio anexo ao Centro de Tecnologia. Rio de Janeiro, RJ, Brasil. CEP 21941-596.

\section{Resumo}

Este artigo discute o papel das terapias hormonais na construção de uma narrativa da saúde para a longevidade da medicina anti-aging. O objetivo é identificar o significado dos hormônios na constituição de um processo de aprimoramento do corpo para manter suas funções naturais e possibilitar a personalização do envelhecimento. A análise é parte da pesquisa realizada no Brasil com médicos praticantes, pacientes e médicos críticos, membros do Conselho Federal de Medicina (CFM), por meio de entrevistas semiestruturadas e observação em campo. Discute-se o estabelecimento de uma rede internacional de profissionais e instituições que viabiliza a expansão das práticas anti-aging em um contexto institucional desfavorável. Na proposição de uma abordagem holística do envelhecimento, a medicina anti-aging se concentra não em doenças típicas da velhice, mas no aprimoramento das condições de saúde ao longo da vida. Considerados "combustíveis" da vida, os hormônios se destacam como elementos de integração entre a particularidade dos pacientes e a generalidade do processo natural de envelhecimento, fortalecendo uma perspectiva de aprimoramento natural, com recursos do próprio corpo. A modulação com hormônios bioidênticos é contraposta à hegemonia da indústria farmacêutica em uma narrativa que altera as fronteiras entre terapia e aprimoramento, natural e artificial, tratamento e estilo de vida.

Palavras-chave: Envelhecimento; Hormônios; Longevidade; Medicina Anti-Aging; Aprimoramento. 
Abstract

This article discusses the role of hormonal therapies in the narrative concerning longevity present in anti-aging medicine. The objective is identifying the meaning that hormones have in the body enhancement process aimed to preserve natural functions and enable the personalization of aging. This analysis is part of a study conducted in Brazil with practitioners of anti-aging medicine, patients and objector members of the Brazilian Council of Medicine, by means of semi-structured interviews and fieldwork observation. The discussion addresses the formation of an international network of professionals and institutions supporting antiaging practices in a disadvantageous institutional context in Brazil. Anti-aging medicine proposes a holistic approach to aging and focuses on enhancing health conditions throughout the patient's life instead of targeting aging-related diseases. Hormones are considered "fuel" for life processes and conceived as elements that can integrate the particularity of each patient and the general aspect of natural tendencies in the aging process. That perspective strengthens the conception of natural enhancement, using the body's own resources. Bioidentical hormone modulation contrasts with the pharmaceutical industry in a narrative that alters the boundaries between therapy and enhancement, natural and artificial, treatment and lifestyle.

Keywords: Aging; Hormones; Longevity; AntiAging Medicine; Enhancement.
Este artigo discute o papel das terapias hormonais na construção da narrativa da saúde para a longevidade da medicina anti-aging. 0 objetivo é identificar o significado dos hormônios na constituição de um processo de aprimoramento do corpo que visa a manter as funções naturais ao longo do tempo e possibilitar a personalização do envelhecimento.

A medicina anti-aging tem uma trajetória que associa diferentes práticas médicas ao objetivo de reverter ou amenizar os sinais físicos do processo de envelhecimento. As fronteiras que a delimitam como prática específica não são evidentes e estáveis, visto que as intervenções passíveis de alterar o envelhecimento surgem de contextos distintos. De elixires de alquimistas a suplementações vitamínicas, diversos experimentos que apontavam a potencialidade de manipular elementos do corpo para alterar os sinais físicos de declínio são associados à história das práticas anti-aging (Olshansky; Hayflick; Carnes, 20o8).

Esse é o caso da organoterapia, que precedeu o desenvolvimento e a institucionalização da endocrinologia e deu ênfase ao uso de substâncias corporais, retiradas de outros animais, para serem utilizadas na alteração de processos fisiológicos. Cientistas como Charles Brown-Séquard, no século XIX, e Serge Voronoff, no século XX, tiveram destaque e reveses em suas carreiras na organoterapia, realizando tratamentos que alegavam reverter sinais do envelhecimento. As práticas destacavam a relevância das substâncias secretadas pelo corpo e os efeitos em seu desempenho. Embora Brown-Séquard tenha sido pioneiro em indicar a função de testículos e ovários como produtores de secreções endógenas e a relação dessas substâncias com as diferenças sexuais, contribuindo para a endocrinologia, teve suas ideias condenadas como charlatanismo, tal como as de Voronoff posteriormente (Cuperschmid, 2014; Tramontano, 2017).

O parâmetro da cientificidade e as acusações de charlatanismo são condições fundamentais do desenvolvimento da medicina anti-aging, sobretudo em sua relação com a esfera institucional da medicina. Desde a ascensão do modelo biomédico, no século XIX, as proposições anti-aging se tornaram periféricas, compreendidas como um objetivo com poucas possibilidades de sucesso devido à 
incompatibilidade com os métodos científicos (Mykytyn, 2007). Entende-se a proposição no sentido adotado por Latour (2004), como afirmação a ser considerada verdadeira ou falsa. Assim, diferentes recursos e estratégias de manipulação do corpo surgiram como caminhos possíveis a serem explorados no combate ao envelhecimento, envolvendo diferentes atores que se articulam em defesa ou contestação de cada um deles. Com o desenvolvimento da endocrinologia, os hormônios ganharam destaque nas explicações sobre o funcionamento do corpo e passaram a ser explorados em diferentes áreas de pesquisa, consolidando-se como um campo de possibilidades para intervenções médicas a ser desvelado. Entretanto, o direcionamento de terapias hormonais para o combate aos efeitos do envelhecimento remete ao estigma do charlatanismo que os empreendimentos anti-aging enfrentam.

A medicina anti-aging se desenvolve mundialmente em um contexto que contrasta o otimismo com as promessas de novos meios de intervenção desenvolvidos por ciências emergentes, como a genética e a medicina regenerativa, e a desconfiança em torno do ideal de controle do processo de envelhecimento. A institucionalização de grupos de profissionais comprometidos com projetos de práticas médicas dedicadas à intervenção no declínio do corpo dá visibilidade e sistematização a esse tipo de abordagem, definindo uma esfera de controvérsias e disputas médico-científicas.

A American Academy of Anti-Aging Medicine (A4M), fundada em 1993, nos Estados Unidos, destaca-se como pioneira na divulgação desse tipo de abordagem e na formação de profissionais especializados. No Brasil, as instituições que buscam por alternativas para lidar com o envelhecimento demarcam oposição entre uma abordagem médica tradicional e a organização de uma forma de medicina que retoma o ideal anti-aging, influenciadas diretamente pela $\mathrm{A} 4 \mathrm{M}$.

A partir do conflito, jurídico e institucional, constituído entre essas instituições e o Conselho Federal de Medicina (CFM), a pesquisa em que este artigo se baseia buscou identificar os vínculos que delineiam a medicina anti-aging no Brasil, considerando sua inserção no contexto internacional.
AAcademia Brasileira de Medicina Antienvelhecimento (ABMAE) foi o ponto de partida para a identificação de associações de elementos que compõem a medicina anti-aging no País. A pesquisa foi feita por meio de entrevistas semiestruturadas com seis médicos do CFM, 14 médicos praticantes da medicina anti-aging e cinco pacientes submetidos aos tratamentos, além de observação em campo em três contextos: (1) Seminário de Geriatria, organizado pelo CFM, no qual foi discutida a situação jurídica da medicina anti-aging no Brasil; (2) curso de formação de médicos ministrado pela ABMAE; e (3) VI Congresso Internacional de Ciências da Longevidade Humana, organizado pelo Grupo Longevidade Saudável. Foram também considerados documentos e publicações dos profissionais acompanhados ao longo da pesquisa. Os diferentes contextos e métodos foram integrados de modo a identificar instituições, porta-vozes, referências e processos que constituem a medicina anti-aging e compreender os fatores de seu desenvolvimento.

A pesquisa tem como base teórico-metodológica a teoria ator-rede (TAR) e a análise foi constituída com foco nas controvérsias, de modo a caracterizar a abordagem específica da medicina anti-aging (Latour, 2004, 2011, 2012). Buscou-se identificar não grupos, mas os processos de formação de vínculos que diferenciam a medicina anti-aging no âmbito da abordagem biomédica do envelhecimento. Nessa conjuntura, os hormônios são o ponto crítico do confronto institucional, evidenciando posicionamentos, ideias e atores que definem o envelhecimento como objeto de tratamento médico.

Latour (2012) ressalta que os contextos de conflito são propícios para a observação do processo de associações e disputas pela delimitação de fatos que organizam a vida em sociedade. 0 mapeamento das controvérsias consiste no acompanhamento dos movimentos temporários e dinâmicos de construção desses fatos. 0 desenho da pesquisa foi definido de modo a acompanhar o processo de recrutamento de aliados e associações que dão forma à rede em que a medicina anti-aging se estabelece e se opõe a outras práticas no contexto da biomedicina. A análise é organizada em três vieses principais: (1) a perspectiva dos médicos praticantes; (2) a percepção dos opositores, sobretudo na condição 
de representantes de instituições médicas oficiais; e (3) o ponto de vista dos pacientes.

\section{O contexto institucional: hormônios e as controvérsias que definem a abordagem anti-aging}

O conflito institucional entre o CFM e os praticantes da medicina anti-aging evidenciou um conjunto de conceitos, práticas e métodos que são associados a essa vertente, estabelecendo uma divisão entre uma abordagem médica oficialmente reconhecida e aquela que não se enquadra nos padrões biomédicos atuais, pela ausência de comprovações por padrões científicos estabelecidos. Em 2012, o Conselho publicou o parecer CFM $n^{0}$ 29/2012, resultante de uma análise realizada pela Câmara Técnica de Geriatria da instituição a respeito do mérito dos tratamentos anti-aging. Essa avaliação foi conduzida a partir da iniciativa do líder do Grupo Longevidade Saudável, que buscava regulamentar as práticas no país, e considerou tanto as referências bibliográficas fornecidas pelo requerente quanto pesquisas em plataformas que reúnem os principais estudos científicos da área biomédica, como MedLine e PubMed. 0 resultado foi desfavorável às práticas anti-aging, resultando em sua condenação pelo CFM por meio da resolução $n^{0} 1.999 / 2012$, documento que proíbe o uso de hormônios, suplementos vitamínicos e sais minerais com o objetivo de retardar ou reverter os sinais físicos e cognitivos de envelhecimento. $\mathrm{Na}$ avaliação do Conselho, não havia evidências consistentes e estudos clínicos suficientes que atestassem que os métodos utilizados promovem a "modulação do envelhecimento saudável".

O parecer e a resolução destacam, dentre as terapias, o uso de hormônios como principal estratégia da abordagem anti-aging, uma vez que são associados à possibilidade de reversão dos principais sinais do envelhecimento, como a perda de massa muscular. Entretanto, a reposição de hormônios para fins sem comprovação de benefícios é considerada perigosa, pois teria diversos efeitos colaterais, incluindo câncer (CFM, 2012a, 2012b).

Os médicos praticantes da medicina anti-aging entrevistados na pesquisa destacam que os hormônios não são a única forma de tratamento que realizam e que, sozinhos, não podem reverter os problemas apresentados pelos pacientes. Por outro lado, reconhecem a potencialidade deles e investem na modulação hormonal como dimensão fundamental de um corpo equilibrado.

A abordagem da medicina anti-aging constituise por meio da oposição crítica ao modelo médico estabelecido, criando uma narrativa da saúde que difere não somente nos tipos de tratamentos propostos, mas na própria concepção de saúde e de envelhecimento. Embora a avaliação do CFM tenha contestado a possibilidade atual de desenvolvimento de uma "modulação do envelhecimento saudável", a narrativa da medicina anti-aging, ao contrário do que se poderia esperar, não se concentra na reversão do processo de envelhecimento em si. A concepção do envelhecimento é fragmentada em processos de saúde e doença de um ponto de vista sistemático, em longo prazo. No lugar de uma perspectiva do envelhecimento como dimensão natural da condição humana, inevitável e com uma lógica própria, a narrativa da medicina anti-aging destaca um processo cumulativo e integrado de condições que levam à manutenção ou à deterioração do organismo. As condições de envelhecimento são o resultado último de outros processos de saúde ao longo da vida. Nessa perspectiva, é possível alterar a forma que se envelhece intervindo sobre condições específicas da saúde.

A ideia de manutenção é associada à de prevenção, defendida como o principal diferencial da abordagem anti-aging. Nesse processo, os hormônios são concebidos como elemento imprescindível, visto que todo o metabolismo depende das interações entre diferentes formas destes para funcionar. Considerando que o declínio hormonal acompanha o avanço da idade, aprimorar os níveis hormonais ao longo da vida permitiria evitar o declínio de diversas funções físicas e cognitivas.

Quando éque na sua vida você deixou de precisar de água? Nunca. Vai precisar de água a té o final e morrer. Assim são os hormônios. [...] Você vê que até hoje o termo andropausa não é considerado. Parece que a única pausa que até hoje os conservadores acreditam é a pausa no ovário feminino. Então eles só falam 
em menopausa. Como se a única coisa que viesse a envelhecer, perder suas funções, no ser humano, fossem os ovários. Então, as mulheres envelhecem nos ovários, nada mais envelhece nas mulheres, nem nos homens. (Médico endocrinologista praticante da medicina anti-aging. Rio de Janeiro, 2015)

A narrativa da medicina anti-aging ressalta um processo de deficiências hormonais, ou "pausas", que não são reconhecidas como patologias pelas instituições médicas, principalmente a endocrinologia. Esse é o caso da "tireopausa" e da "fadiga adrenal", conceitos que delimitam condições causadas por deficiências na produção de hormônio pelas glândulas tireoide e suprarrenal, respectivamente. Observa-se a ênfase nos hormônios como substâncias capazes de desequilibrar os indivíduos, tanto do ponto de vista físico e cognitivo quanto comportamental.

A natureza é assim: de 20 a 30 anos ela te mantém $100 \%$. Por quê? Porque ela quer que você reproduzae que você mantenha a sua espécie. O nosso objetivoé manter a espécie. Reproduzir e morrer. Só que a gente insiste, não é? A gente continua vivendo. Então, o que vai acontecendo? A partir dos 30 você vai perdendo várias substâncias, mas principalmente a hormonal, e depois dos 50 você despenca. Então, o que a gente procura fazer? É manter exatamente igual quando você tinha de 20 a 30 anos. Então a referência que o laboratório vai me colocar, ele vai colocar aqui o valor normal, por exemplo, se eu coloco do DHEA [desidroepiandrosterona] ou da testosterona, ele vai estar me dando por faixa etária o valor normal, ou seja, ele vai estar aceitando esse processo fisiológico de perda que todo mundo vai passar. Eu não vou aceitar isso aqui na anti-aging. (Médica nutróloga praticante da medicina anti-aging. Curitiba, 2017)

Ao analisar os usos do hormônio do crescimento desde a sua disponibilização e aprovação pelo Food and Drugs Administration (FDA), dos Estados Unidos, em 1985, Conrad e Potter (2004) destacaram as controvérsias em torno de sua função e dos tipos de terapias possíveis. Indicado originalmente para tratar pessoas com deficiência na produção endógena, sobretudo crianças com crescimento abaixo dos níveis considerados normais, o hormônio foi investigado do ponto de vista dos seus efeitos para outros usos. Os autores discutem os limites entre a realização de terapias com hormônio do crescimento para fins de reparação da deficiência e o uso para aprimoramento estético e de desempenho físico. Nesse sentido, o desenvolvimento da produção sintética do hormônio, na década de 1990, tornou o acesso a esse recurso mais fácil, alterando os limites aceitos como legítimos pelas instituições médicas e aumentando os usos off-label.

Um importante aspecto destacado pelos autores diz respeito ao processo de ampliação da concepção de uso terapêutico legítimo associado a características que são valorizadas e relevantes de um ponto de vista social. No caso das crianças com baixa estatura, nem sempre essa condição está relacionada com a deficiência na produção de hormônio do crescimento. 0 parâmetro considerado passa a ser a estatura em si, quando esta representa um desvio dos padrões. Embora cada população tenha uma média considerada normal, há sempre indivíduos nos extremos, muito altos ou muito baixos. Entretanto, o estigma da baixa estatura, principalmente para os homens, conduz à aceitação da utilização do hormônio como terapia. É seu potencial efeito sobre o crescimento dos indivíduos, associado a uma expectativa social sobre a estatura ideal, que permite uma equiparação das duas condições, com e sem a deficiência do hormônio, como alvos da intervenção médica.

A identificação e o aprofundamento sobre os processos biológicos que sofrem influência de determinado hormônio geram especulações que favorecem experiências de usos alternativos. No caso do hormônio do crescimento, os estudos que apontavam sua influência em processos somáticos levaram à sua indicação não apenas para o crescimento, mas também para a recuperação muscular e o melhoramento do desempenho físico, o que atraiu principalmente atletas.

O surgimento de diversas terapias com esse hormônio incitou divergências e promoveu a reorientação de processos regulatórios que, na perspectiva da cientificidade e da minimização dos riscos na prática médica, visa a limitar o uso dessas substâncias a determinadas condições com 
procedimentos institucionalizados. O interesse criado em torno da potencialidade dos efeitos do uso de hormônios, todavia, estabelece redes de interesses que se opõem nesse processo de regulação, envolvendo diferentes atores, como políticos, laboratórios e entidades médicas. Nessa conjuntura, os profissionais, sobretudo os médicos a quem é delegada a função do diagnóstico e da determinação terapêutica adequada, têm uma posição estratégica que os permite transitar entre as instâncias do conflito.

Os praticantes da medicina anti-aging têm em comum o interesse por uma prática médica que se opõe a parâmetros e práticas estabelecidos, buscando por alternativas aos tratamentos convencionais. O modelo médico oficial é considerado impessoal, mecanicista e fragmentado, impedindo uma abordagem que identifique fatores iniciais que levam às disfunções do corpo ao longo do tempo. A proposição de uma medicina holística e não curativa estabelece o aprimoramento constante do corpo como um objetivo da prática médica, aproximando a dimensão do enhancement da reparação e restituição da normalidade. Por meio dessa narrativa da saúde, os hormônios se destacam como instância estratégica para o desenvolvimento de uma abordagem centrada na funcionalidade e no desempenho do corpo ao longo do tempo como meio de alterar o declínio no envelhecimento. Como entidades que têm suas propriedades definidas nesse processo de controvérsias sobre os benefícios e efeitos colaterais das terapias, os hormônios moldam também os vínculos estabelecidos entre os diferentes atores que compõem a medicina anti-aging no Brasil.

Dadas as limitações institucionais, os praticantes da medicina anti-aging associamse a diversas áreas de conhecimento, como medicina esportiva, medicina ortomolecular, nutrição e estudos de fisiologia hormonal. Por meio de parcerias, eventos e cursos de formação, as instituições dedicadas às práticas anti-aging organizam uma rede de apoio e troca de informações e recursos, com atores nacionais e internacionais. Essa rede viabiliza a especialização de profissionais e a realização de diagnósticos e tratamentos, como é o caso de testes genéticos e da suplementação com substâncias não produzidas ou não regulamentadas para venda no Brasil.

Um importante aspecto da medicina anti-aging é que ela não é praticada por atores externos às instituições biomédicas oficiais, como uma forma terapêutica alternativa. São profissionais formados em instituições tradicionais, que fazem parte das sociedades médicas de especialidade oficiais. Esses cardiologistas, cirurgiões plásticos, endocrinologistas e nutrólogos, todavia, apresentam uma prática médica comum, que sobrepõe suas especialidades tradicionais.

No Brasil, as instituições ABMAE e Grupo Longevidade Saudável lideram a formação de profissionais e a organização de eventos. Muitos profissionais que se destacam criam suas próprias empresas, nas quais promovem palestras e cursos de formação em práticas específicas, expandido o alcance da narrativa anti-aging. A Sociedade Brasileira para Estudos da Fisiologia (Sobraf), presidida pelo líder do Grupo Longevidade Saudável, destaca a relevâncias dos hormônios nessa proposição de medicina. Nas relações estabelecidas com as instituições internacionais, destacam-se a $\mathrm{A}_{4} \mathrm{M}$ e o Cenegenics Education and Research Foundation - Age Management Medicine, além da World Society of Anti-Aging Medicine (WOSAAM) e da International Hormone Society, ambas lideradas pelo médico belga Thierry Hertoghe.

Entre os médicos pesquisados, o uso do termo anti-aging ou antienvelhecimento apresenta ambiguidades. Há um desconforto em utilizar os termos e fazem ressalvas a respeito do seu sentido verdadeiro, afastando a ideia de busca pela juventude eterna. Os médicos, principalmente depois da publicação da Resolução ${ }^{0}{ }^{1}$ 1.999/2012 pelo CFM, são proibidos de utilizar o termo anti-aging ou antienvelhecimento como modalidade médica. Entretanto, em publicações e descrições em páginas na internet e materiais de divulgação desses profissionais, a referência ao anti-aging aparece como forma de distinção.

O termo anti-aging é, mais do que um nome, um conceito que incorpora a trajetória de controvérsias em torno do objetivo de alterar o envelhecimento. Ele é parte relevante das associações que definem 
a ciência do envelhecimento, situando práticas específicas e definindo fronteiras na abordagem biomédica. Optou-se por utilizar o termo antiaging por refletir o contexto de controvérsias no desenvolvimento das práticas analisadas.

Durante o VI Congresso de Ciências da Longevidade Humana, um dos principais eventos da área, realizado no Brasil pelo Grupo Longevidade Saudável, na cidade de São Paulo, foi possível identificar, de forma sistemática, a correlação entre o potencial dos hormônios e o objetivo de combater o envelhecimento. A maioria das conferências dos três dias de evento abordava os variados usos possíveis de hormônios. Dentre os palestrantes estavam profissionais de destaque na realização de terapias hormonais, que relatavam suas experiências e discutiam os melhores meios e técnicas para o processo terapêutico. A possibilidade de controlar o envelhecimento permeava todo o debate de forma explícita, pois o aprimoramento do corpo em diferentes aspectos é orientado a evitar a degradação que acompanha o avanço da idade. Essa perspectiva de intervenção é marcada pela proposição de reconstrução constante dos processos naturais do corpo - uma extensão do funcionamento ideal do organismo, que, todavia, contrapõe o declínio como processo necessário do curso natural da vida. Essas intervenções são direcionadas para a composição de um novo estilo de vida.

\section{Metabolismo, estilo de vida $e$ fisiologia hormonal: totalidade $e$ particularidades}

$\mathrm{Na}$ perspectiva da medicina anti-aging, os hormônios são combustíveis que podem ser utilizados para evitar o declínio. Entretanto, a fisiologia hormonal é concebida como um processo integrado e sua manutenção depende de que as partes funcionem em harmonia. Nesse sentido, os tratamentos com hormônios não se limitam à reposição. A noção de modulação hormonal expressa a proposição de uma medicina holística, com uma abordagem da saúde que integre diferentes processos que concorrem para a determinação de um estado de saúde ou doença.
Um dos principais conceitos da narrativa da saúde na medicina anti-aging é o metabolismo que, como um sistema, depende de cada uma de suas partes para funcionar plenamente. Uma vez que o envelhecimento é percebido como resultado do acúmulo de falhas e insuficiências, é a manutenção dos processos metabólicos que torna possível evitar o declínio ao longo dos anos. Ao mesmo tempo que o metabolismo constitui a materialidade da vida, ele estabelece uma constante interação com o meio, sendo influenciado por fatores externos e pela conduta dos indivíduos. A principal estratégia anti-aging seria manter o corpo com os nutrientes e hormônios necessários às funções básicas e retirar elementos danosos ao organismo, restabelecendo as condições ideais e naturais de seu funcionamento.

Uma vez que a quantidade e o tipo de danos acumulados variam de acordo com as condições de vida individuais e as mudanças biológicas não necessariamente acompanham o avanço da idade, haveria diferentes níveis de envelhecimento. Assim, uma pessoa mais velha cronologicamente pode ser mais nova biologicamente. O metabolismo favorece a ênfase na individualidade das condições de envelhecimento na medida em que articula processos naturais universais com a particularidade do contexto vivenciado por cada pessoa. Nessa mesma lógica, estabelece-se a orientação para a reorganização do estilo de vida, com a adaptação de hábitos individuais, como alimentação, atividades físicas e controle de estresse, para garantir as condições de vida favoráveis ao corpo.

A modulação hormonal manifesta essa integração na medida em que se baseia no equilíbrio de toda a fisiologia hormonal, dependendo da nutrição, do condicionamento físico e do controle de emoções.

Não adianta também eu me preocupar, fazer a modulação hormonal num paciente que não tem uma alimentação saudável, que não pratica um exercício e que é irritado e mal-humorado. Não vou ter também um resultado tão bom. O hormônio não vaifazer esse milagre. (Médica nutróloga praticante da medicina anti-aging. Curitiba, 2017)

A concepção da modulação hormonal como processo multifatorial pode ser associada à 
influência da perspectiva ortomolecular na abordagem anti-aging. Linus Pauling (1901-1994) é considerado pioneiro dessa prática e responsável por cunhar o termo ortomolecular, o qual expressa a concepção do corpo humano como uma estrutura de moléculas. Pauling (1978, p. 4, tradução nossa) defende a ideia de que é necessário "ter as moléculas certas nas quantidades certas" para ter saúde e uma vida longa.

Os processos bioquímicos interdependentes do metabolismo necessitam da quantidade e do tipo correto de molécula para a produção de elementos imprescindíveis às funções corporais. Assim, a prática terapêutica elaborada por Pauling consiste em prover os recursos necessários para o desenvolvimento saudável do corpo e eliminar o que interfere nas reações bioquímicas corretas. Essa abordagem dá ênfase ao processo de interação dos indivíduos com o meio, destacando o contato com substâncias e a alimentação como processos determinantes da composição corporal.

A prática da modulação hormonal exige, portanto, uma reeducação dos pacientes, na medida em que implica uma revisão de condições de vida para viabilizar os efeitos esperados. Essa forma de intervenção vai além da dinâmica de um tratamento conduzido de maneira pontual pelo profissional de saúde, dependendo principalmente das ações do próprio paciente.

Um dos principais diferenciais da abordagem da medicina anti-aging é a relação estabelecida entre médicos e pacientes. Os profissionais têm em comum uma trajetória em que eles mesmos se submetem aos tratamentos e ao estilo de vida proposto, servindo de exemplo aos pacientes. Essa prática faz com que estabeleçam um vínculo por meio do compartilhamento do estilo de vida.

A medicina anti-aging retrata as mudanças físicas e cognitivas que acompanham o envelhecimento como um gradativo afastamento das condições necessárias para o desenvolvimento de habilidades e capacidades. 0 declínio inviabiliza a individualidade, na medida em que as limitações fazem com que as pessoas deixem de fazer aquilo que gostavam e que caracterizava sua trajetória particular. A essência individual se perde no processo de se tornar um "velho" ou "velha”, condição genérica marcada pela incapacidade. As intervenções, concebidas como um processo de recuperação e manutenção, não são vistas como algo artificial, que modifica a condição natural dos indivíduos: a "medicina do estilo de vida" define as intervenções mais como um cuidado de si do que como um tratamento médico. 0 uso de hormônios, associado às mudanças de conduta, é apresentado como uma forma de recuperação das funções naturalmente estabelecidas, permitindo que as pessoas mantenham suas atividades.

Para os pacientes, as terapias são uma opção para lidar com o próprio envelhecimento. Observa-se que os pacientes pesquisados compartilham com os médicos as críticas ao modelo médico convencional, em relação ao qual destacam a incapacidade de solucionar os sofrimentos, muitas vezes sem diagnóstico, que se tornavam um obstáculo às trajetórias pessoais.

Aí fui a uma endócrino. Três endócrinos. Três. Aí um olhou: "Não, não tem nada. Você está excelente." "Não é a tireoide, doutor? Não tem nada, nenhuma disfunção?" "Não, na sua idade você está ótima!". Caramba! Então ser ótimo para um médico tradicional é ser depressiva, gorda, com enxaqueca. Só faltou eu falar "Querida, eu estou a um passo de me suicidar! Eu estou morrendo na praia! A minha vida inteira eu fui atleta, agora eu estou $10 \mathrm{~kg}$ mais gorda, com enxaqueca há dez anos e sem um médico descobrir o que é! Evocês falando que eu estou bem. Que droga de medicina é essa?!". (Mulher educadora física e coach de carreiras, 56 anos. São Paulo, 2017)

Todos os pacientes pesquisados afirmaram ter ciência das controvérsias envolvendo os tratamentos a que estavam tendo acesso e da reprovação dessa forma de medicina pelo CFM e demais instituições reguladoras da saúde. Todavia, por serem críticos do modelo convencional de medicina, esse fator se tornava uma vantagem e motivo de buscarem esse tipo de tratamento. Os pacientes veem nessa prática médica a possibilidade de darem um significado pessoal ao tratamento, na medida em que se percebem atuando de forma ativa nesse processo.

Eu comecei, então, a sair dessa bolha de acreditarno que qualquer revista ou qualquer jornal na televisão 
falava para mim e comecei a entender mais sobre artigos, eu lia. Por exemplo, ele vai lá e fala bem do leite. Eu vou ler todas as meta-análises, estudos recentes e antigos que saíram sem patrocínio da indústria do leite, e você vê que não bate. Você vê que ele está sendo pago para falar aquilo. Então esse caminho que eu segui para conhecer $o$ anti-aging, para conhecer o que é bem-estar de verdade, o que é você estar com o equilíbrio hormonal, ciclo circadiano. (Homem ator e empresário, 30 anos. Rio de Janeiro, 2017)

Os pacientes não veem os tratamentos como uma busca da juventude eterna, mas como uma forma de cuidarem de si para manter as condições físicas e cognitivas e dar continuidade a seus projetos de vida. Abordam o tratamento como mudança de atitude para terem mais saúde, em oposição a um modelo médico anterior em que eles ficariam restritos a medidas reativas para sintomas específicos. Na lógica da "medicina do estilo de vida", os pacientes descrevem um processo de reeducação e consciência de si, em que os hábitos cotidianos passam a ser interpretados em termos de consequências em longo prazo. O uso de suplementos e de hormônios é contraposto à dependência de medicamentos, percebidos como um recurso artificial.

Nikolas Rose (2011) apresenta o conceito de self como ideal regulatório, constituído por meio de regimes de subjetivação em que a percepção de si e a formação de uma ética de autoavaliação são elaboradas por meio de um aparato de técnicas e tecnologias específicas. Nas sociedades contemporâneas, as regulações que fazem parte desse processo são, sobretudo, realizadas por discursos especialistas, que transformam questões da experiência de vida em questões técnicas quanto a formas mais eficientes de gerenciar a qualidade de vida. A constituição do self é historicamente situada, refletindo valores e condições políticas e econômicas que caracterizam uma época. Dessa forma, a prevalência de valores como autonomia, liberdade, independência e a responsabilização dos indivíduos pela sua autorrealização fazem com que o declínio esperado do envelhecimento contraste com o ideal de pessoa baseada na individualidade e particularidade da trajetória.

No contexto de promoção do envelhecimento ativo, observa-se uma condição ambígua. Mais do que um parâmetro para políticas públicas, o envelhecimento ativo se tornou um processo de educação, preparação e adaptação para o envelhecimento (António, 2015). Assim, há um contraste entre um processo natural, em que o declínio físico e cognitivo é parte das expectativas, e o chamado à condução do próprio envelhecimento para se manter ativo. Os pacientes apresentam uma preocupação comum de reagir contra o desgaste do corpo para manter suas personalidades. Ao destacar as condições individuais do processo de envelhecimento e explorar a possibilidade de moldar a forma de envelhecer de acordo com as trajetórias pessoais, a medicina anti-aging atua sobre essa condição ambígua vivenciada pelos pacientes. Por se afastar de uma abordagem da velhice, ela tem a vantagem de favorecer que esses pacientes lidem com as intervenções em longo prazo como um cuidado de si para se adaptar ao processo de envelhecimento. A ênfase na noção de estilo de vida dialoga com a tendência de superação da perspectiva dicotômica entre saúde e doença na biomedicina contemporânea (Rose, 2013). É a qualidade da vida em si, em termos de desempenho e funcionalidade, que estabelece os parâmetros de normalidade e saúde.

Embora os pacientes afirmem que o tratamento exige muita dedicação, pois são muitas mudanças e substâncias para serem administradas, ele é percebido como uma recomposição de elementos naturais, de algo que faz parte do que são, afastando a ideia de que estão com falhas que precisam ser controladas. Do mesmo modo, na perspectiva dos profissionais, a modulação hormonal é uma forma de romper com tratamentos com remédios, pois com o equilíbrio do corpo as condições que levam à necessidade de utilizá-los seriam evitadas. Contrapondo substâncias artificiais, são os próprios recursos do corpo que devem ser utilizados como uma ferramenta de aprimoramento do bem-estar e da personalidade. Se o corpo perde, naturalmente, esses elementos, é proposta uma intervenção: a reprodução da natureza. 


\section{Bioidênticos: a reconstituição da} lógica da natureza

Uma das principais esferas em que o conflito entre praticantes da medicina anti-aging e o CFM se desenvolve é a econômica e/ou mercadológica. Interesses financeiros envolvidos na prática médica emergem como um fator de disputas em torno da cientificidade e da ética dos tratamentos que são disponibilizados aos pacientes. 0 debate inclui o processo em que os procedimentos médicos são testados, avaliados e liberados. Os critérios de cientificidade são questionados por ambos os lados e o fator econômico se destaca como parâmetro utilizado para acusações mútuas. Nesse conflito, são associados diferentes atores que se alinham em grupos opostos, evidenciando a rede que delimita e diferencia as práticas anti-aging de um modelo oficial de medicina. Destaca-se, nesse contexto, a tentativa de afastamento da medicina anti-aging das grandes indústrias, identificadas como responsáveis pela artificialidade danosa do estilo de vida moderno.

$\mathrm{Na}$ medida em que a narrativa da medicina anti-aging se constitui em oposição e crítica às práticas e parâmetros consolidados na biomedicina, há uma vinculação direta entre as limitações da regulação sobre acesso e usos de recursos e procedimentos e os interesses de diferentes setores com influência sobre a prática médica, o que é o caso principalmente da indústria farmacêutica. O CFM é acusado de representar, institucionalmente, os interesses desses atores. Ainda que essa indústria tenha desempenhado um papel fundamental na popularização de usos off-label de medicamentos, inclusive de hormônios (Azize, 2002; Rohden, 2011), ela é associada às limitações impostas aos tipos de terapias hormonais que são oficialmente reconhecidas por controlar os processos de experimentos clínicos e regularização das substâncias.

A associação com o charlatanismo molda o status institucional e a condição dos profissionais na comunidade médica. Observa-se um esforço de contraposição não apenas às alegações de falta de comprovação de benefícios dos usos de hormônios, mas, principalmente, às concepções que definem a legitimidade desses tratamentos. 0 apelo à natureza estabelece uma estratégia retórica em que o natural não pode ser mais perigoso do que muitos usos artificiais já aceitos pelas autoridades em saúde. Esse é o caso do uso de contraceptivos hormonais, amplamente criticado e condenado pelos praticantes da medicina anti-aging. Abordados como "castração química" de mulheres, os contraceptivos são explorados como evidência de que os parâmetros utilizados para a liberação de usos não são definidos somente com base na eficácia e nos riscos para a saúde de pacientes, mas envolvem interesses sociais e financeiros. Os médicos destacam que esses medicamentos têm sido usados inclusive para tratamentos não relacionados à dimensão reprodutiva, sendo prescritos para problemas de pele, inclusive para adolescentes, a despeito dos inúmeros efeitos colaterais da alteração da fisiologia hormonal.

No parecer-consulta do CFM, o uso do termo "bioidêntico" para diferenciar as terapias hormonais na medicina anti-aging é alvo de contestação. A associação é considerada mera ação de marketing, pois os hormônios disponibilizados pela indústria farmacêutica são fabricados para se comportarem exatamente como os hormônios produzidos no organismo. Não haveria, portanto, nenhuma diferença que justificasse a distinção.

Muito se fala, hoje, dos chamados "hormônios bioidênticos", substâncias hormonais que possuem exatamente a mesma estrutura química e molecular encontrada nos hormônios produzidos no corpo humano. Esta nomenclatura está sendo utilizada indevidamente apenas para os hormônios manipulados, como se fossem novas opções de tratamento, quando, na verdade, há muito tempo hormônios bioidênticos são produzidos em indústrias farmacêuticas e estão disponíveis nas farmácias. (CFM, 2012b)

Como a própria crítica apresentada pelo CFM destaca, a narrativa da medicina anti-aging busca afastar elementos ou práticas que identifiquem maior artificialidade, utilizando esse aspecto para contrapor o modelo médico oficial. 
Rivotril está o número um em vendas aqui no Brasil. [...] A indústria farmacêutica não é interessada em que você compre melatonina. Melatonina não lhe vicia, melatonina não lhe dá efeito colateral, você não tem que aumentar dose, ela é natural. Ela não pode ser patenteada, assim como nenhum outro hormônio. (Médico clínico geral praticante da medicina anti-aging. Rio de Janeiro, 2015)

A concepção de hormônio bioidêntico é associada pelos praticantes da medicina antiaging não apenas à sua composição, mas também à sua produção para uso médico e à forma de aplicação. Esses profissionais evitam o uso de medicamentos produzidos em larga escala pela indústria farmacêutica, recorrendo principalmente às farmácias de manipulação. O processo manufaturado é relacionado à possibilidade de personalização do tratamento e maior controle do profissional que faz a prescrição. As patentes são vinculadas à necessidade de alterar, ainda que minimamente, a estrutura dos hormônios comercializados, pois não seria possível patentear algo feito pela natureza. Assim, a manufatura possibilitaria acesso direto às substâncias que, embora fabricadas, podem reproduzir a estrutura original.

As principais formas de terapia hormonal são por via transdérmica, em gel ou creme, e por meio de pequenos chips ou pellets implantados sob a pele. Busca-se um meio ideal de reprodução, o mais fiel possível, da dinâmica natural da fisiologia hormonal. Os implantes hormonais, nesse sentido, são apresentados como uma evolução dos métodos, sendo o meio mais eficiente por não depender da ação do paciente, evitando influência externa em seu funcionamento. Como mostram Manica e Nucci (2017), o aprimoramento tecnológico na manipulação de hormônios com o uso de implantes e microchips é associado à possibilidade de personalização de processos da fisiologia hormonal. A artificialidade dos tratamentos hormonais com interesses diversos é contraposta pela ideia de uma intervenção adaptada às condições naturais do corpo, o que reduziria incômodos e efeitos colaterais.
A noção de bioidêntico utilizada na elaboração das terapias hormonais se associa às dimensões do metabolismo e da estratégia do estilo de vida para compor uma prática médica que remete o paciente à manutenção de sua natureza, ao mesmo tempo retomando uma ordem universal e reconstituindo as particularidades de cada organismo e de cada trajetória com objetivos específicos.

\section{Considerações finais}

A abordagem da medicina anti-aging na contemporaneidade é elaborada a partir da própria condição controversa historicamente estabelecida no âmbito das tentativas de identificar meios de controlar o processo de envelhecimento. Desse modo, sua desvantagem em relação aos parâmetros de cientificidade que legitimam as instituições biomédicas, e que são por elas regulados, se torna uma vantagem estratégica, na medida em que ela explora as contradições da experiência de envelhecer. A perspectiva biomédica hegemônica que destaca o caráter natural e inevitável do declínio do envelhecimento é vivenciada pelos pacientes como um obstáculo às exigências e expectativas de uma vida cada vez mais longa.

O paradigma do envelhecimento ativo estabelece, paradoxalmente, um contexto favorável ao fortalecimento das ideias anti-aging devido à ambiguidade da perspectiva de que é necessário se preparar para envelhecer, mas aceitar o envelhecimento como processo inevitável, que deve ser vivido para além da mera condição física. A medicina anti-aging explora esse contraste, propondo uma revisão da concepção do envelhecimento. A ênfase nos processos metabólicos como definidores, em longo prazo, das condições do envelhecimento favorece uma abordagem médica que se afasta da ideia de cuidados específicos na velhice e permite aos pacientes pensarem o processo de tratamento como um cuidado de si para resistir aos efeitos da passagem do tempo e dar continuidade a suas trajetórias pessoais.

A medicina do estilo de vida, por meio da modulação hormonal, reorienta a relação dos pacientes com as práticas biomédicas para a 
constante preocupação com a qualidade das condições de vida. Por meio do aprimoramento de si, o processo de envelhecimento é percebido por profissionais e pacientes como a possibilidade de vivenciar o envelhecimento de forma particularizada, distanciando-se de uma experiência genérica de tornar-se um "velho" ou "velha".

A adaptação das intervenções médicas à reprodução das condições naturais de vida expressa a ressignificação da noção de anti-aging ou antienvelhecimento, em que se pretende que seja não uma oposição a envelhecer, mas uma mudança na forma em que se envelhece. Como "medicina da longevidade saudável", condiciona a possibilidade de um bom envelhecimento à manutenção da funcionalidade do corpo, tornando possível a personalização desse processo. Como combustíveis que mantêm a máquina do corpo em funcionamento, os hormônios se estabelecem como o meio privilegiado de reconstituir as naturezas particulares.

\section{Referências}

ANTÓNIO, M. A. S. H. C. Envelhecimento ativo e o recurso à medicina tradicional chinesa: entre a responsabilidade individual e os fatores sociais determinantes da saúde. 2015. Tese (Doutorado em Antropologia) - Universidade de Lisboa, Lisboa, 2015.

AZIZE, R. A química da qualidade de vida: um olhar antropológico sobre o uso de medicamentos e saúde em classes médias urbanas brasileiras. 2002. Dissertação (Mestrado em Antropologia Social) - Universidade Federal de Santa Catarina, Florianópolis, 2002.

CONRAD, P.; POTTER, D. Human growth hormone and the temptations of biomedical enhancement. Sociology of Health \& Illness, Chicago, v. 26, n. 2, p. 184-215, 2004.

CFM - CONSELHO FEDERAL DE MEDICINA. Processo-consulta CFM $n^{\circ}$ 4.69o/11: Parecer CFM no 29/2012. Brasília, 2012a. Disponível em: <https://bit.ly/39x1JFq>. Acesso em: 15 nov. 2019.
CFM - CONSELHO FEDERAL DE MEDICINA.

Resolução $n^{0}$ 1.999, de 19 de outubro de 2012. A falta de evidências científicas de benefícios e os riscos e malefícios que trazem à saúde não permitem o uso de terapias hormonais com o objetivo de retardar, modular ou prevenir o processo de envelhecimento. Diário Oficial da União, Brasília, DF, p. 139, 19 out. 2012b. Seção 1. Disponível em: <https://bit.ly/2uTITJO>. Acesso em: 15 nov. 2019.

CUPERSCHMID, E. M. O doutor Voronoff de Mendes Fradique. WebMosaica, Porto Alegre, v. 6, n. 2, p. 112-123, 2014.

LATOUR, B. Políticas da natureza: como fazer ciência na democracia. Bauru: Edusc, 2004.

LATOUR, B. Ciência em ação. São Paulo: Editora Unesp, 2011.

LATOUR, B. Reagregando o social: uma introdução à teoria do ator-rede. Salvador: Edufba, 2012.

MANICA, D.; NUCCI, M. Sob a pele: implantes subcutâneos, hormônios e gênero. Horizontes Antropológicos, Porto Alegre, n. 47, p. 93-129, 2017. Disponível em: <https://bit.ly/38lFaT1>. Acesso em: 22 jan. 2020.

MYKYTYN, C. E. Executing aging: an ethnography of process and event in anti-aging medicine. 2007. Tese (Doutorado em Antropologia) - University of Southern California, Los Angeles, 2007.

OLSHANSKY, S. J.; HAYFLICK, L.; CARNES, B. No truth to the fountain of youth. Scientific American, Palmetto Bay, dez. 2008. Disponível em: <https://bit.ly/32RbPP1>. Acesso em: 21 jan. 2020.

PAULING, L. Orthomolecular enhancement of human development. In: HUMAN NEUROLOGICAL DEVELOPMENT: PAST, PRESENT, AND FUTURE: a joint symposium sponsored by NASA/Ames Research Center and the Institutes for the Achievement of Human Potential, 1978, Moffett Field. Proceedings... Moffett Field: NASA CP, 1978.

ROHDEN, F. "O homem é mesmo a sua testosterona”: promoção da andropausa e representações sobre sexualidade e 
envelhecimento no cenário brasileiro.

Horizontes Antropológicos, Porto Alegre,

v. 17, n. 35, p. 161-196, 2011.

ROSE, N. Inventando nossos selfs: psicologia, poder e subjetividade. Petrópolis: Vozes, 2011.

ROSE, N. A política da própria vida: biomedicina, poder e subjetividade no século XXI. São Paulo: Paulus, 2013.

TRAMONTANO, L. Testosterona: as múltiplas faces de uma molécula. 2017. Tese (Doutorado em Saúde Coletiva) - Universidade do Estado do Rio de Janeiro, Rio de Janeiro, 2017. 